\title{
REVIEW OF CAD VISUALIZATION STANDARDS IN PLM
}

\author{
Stephane NZETCHOU ${ }^{1 *}$, Alexandre DURUPT ${ }^{1}$, Sebastien REMY², Benoit \\ EYNARD $^{1}$ \\ ${ }^{1}$ Université de Technologie de Compiègne, Laboratoire Roberval FRE CNRS 2012 \\ Rue du Dr Schweitzer, 60203 Compiègne Cedex, France \\ ${ }^{2}$ Université de Technologie de Troyes, Institut Charles Delaunay LASMIS FRE CNRS 2848 \\ 12 rue Marie Curie, 10010 Troyes Cedex, France

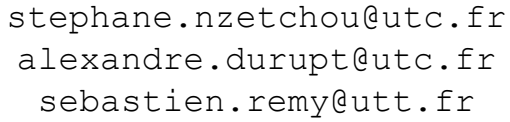

\begin{abstract}
The rise of new technologies has led to a growth in the number of 3D data. More 3D data are voluminous, greater the number of interrelationships between the data. These data can come from various sources, hence their heterogeneity and complexity. The level of data access is often a function of the user's expertise since the 3D data are complexes and registered to different file formats. A 3D data file format is used for storing information about 3D models. Each sector adopts his own 3D file format for different reasons. In this article, we are going to learn about 3D file formats and survey the functionality differences between some popular file formats, able to ease the integration of data, by analyzing on $3 \mathrm{D}$ viewing technology, some models made by two different $\mathrm{CAD}$ systems.
\end{abstract}

Keywords: PLM, 3D modelling, File formats, Data exchange, Application Interoperability

\section{Introduction}

The way companies communicate, has changed. Companies send most of information digitally instead of paper and it is considered as a great forward in saving time and money. One of the ways to save money is the business mindset of Product Lifecycle Management (PLM).

Inside the PLM system, the data from which the information is extracted, are heterogeneous by their type (photo, video, text file, CAD file, etc.); the CAD data can be heterogeneous by their formats: legacy formats (CATIA, CREO, NX, etc.), neutral formats (STEP, JT, IGES, STL, etc.), but also from the expertise that created the data or which it is intended for. Thus, the links and the dependences, which appear between heterogeneous $\mathrm{CAD}$ data, are becoming more complex during the daily activities of

adfa, p. 1, 2011.

(C) Springer-Verlag Berlin Heidelberg 2011 
users. When products are developed in 3D for the engineering domain, the data are initially stored in the legacy file format of the used CAD software. If this 3D CAD data is to be made available to people who do not have this software, neutral 3D formats are needed. Some file formats are: 3D PDF from Adobe, X3D, U3d from Web3D consortium, JT and STEP [1]. The proprietary nature of CAD vendor specific data limit the possibility of open-ended analysis of the 3D data and creates restricted universality of such tools since they work only for the format or API of the particular application [2]. The access to the information becomes a laborious task due to the non-structuring of the data and the lack of sharing information. "How can we overcome dependence on legacy formats in order to access geometric and topological information? How can we allow any user to query and retrieve geometric and topological information to a desired granularity?" This raises the need to analyze 3D neutral formats to see their distinctive features view and PMI (product \& manufacturing information).

\section{Product Lifecycle Management}

PLM is made to be composed of digital product definition and delivery. The tasks are based on computer models processed with computer assistance. According to Terzi [3], most of the product data are generated at beginning of life (BOL). The software owned by a company, particularly 3D CAD systems, have to be able to communicate with each other. This type of tool is developed to help product designers and to provide a traceability support of the evolution of an artefact from the requirements to the product use and even recycling. Digital product definition forms the origin and core of PLM. Product definition evolved from engineering drawings to computer-aided $\mathrm{X}(\mathrm{CAx})$ as said in [4]. Most of CAD systems enable exchange of the shape models with CAx (CAE, CAPP, CAM etc.) systems. Tools are being developed to optimize the design subject to conflicting requirements in one pass. This enables designers to release physical testing and simulation to digital simulation and prototyping for much of the design.

For PLM being successful, data formats of digital product must be able to convey design intent, be machine interpretable, and lose as little information as possible in the translation process. From McHenry [5], some problems arisen within companies having different file formats and due to format conversions and companies outsourcing are: Missing, collapsed, inverted faces, models that do not form closed solids (surfaces and edges do not connect). The major at hand is the interoperability between 3D CAD systems. Companies can have an issue even in-house if multiple 3D CAD systems are used.

\section{Research background of some interoperable 3D file format}

\subsection{Studies of interoperable files}

Research Triangle Institute for the National Institute of Standards and Technology has performed a research to determine the differences of STEP and lightweight file formats in 1999. This study analyzed the transfer of models from one CAD package to 
another by way of STEP and an outdated standard, IGES [6]. It was very simple and maybe effective for 1999, but technology, the standard, and other variables have improved and changed since then. More recent studies performed on lightweight file formats like lightweight Formats for Product Model Data Exchange and Preservation [7]. However, this study only covers the basics of model fidelity, metadata support, security, features, file size, software support, and it is important since it compares basis differences between many lightweight formats. Academia has performed studies to try and to evaluate the difference between STEP, JT, 3DXML, and U3d. This study was performed to demonstrate all the difference regarding the functionality of part registered inside these formats. [8]. ProSTEP iViP Association is an international association that helps drive the development of vendor-neutral file standards and validates the quality of software solutions for interoperability. ProSTEP performs research on benchmarking STEP and JT. ProSTEP published 3D Formats in the Field of Engineering, a Comparison in 2010, in which JT, STEP, and 3D PDF were analyzed using multiple CAD systems and several attributes. The CAD systems used were CATIA V5, CREO, and Siemens NX [9].

It is clear that an in-depth multi-level comparison should be done with the STEP standard and other new coming file formats as JT because the design in context is one of the most processes which could be based on JT and structure information (STEP AP242XML[10] - figure 2) coming from various 3D CAD systems. The latter two file formats include the annotations and attributes associated with the edges and faces of CAD model in order to detail product geometry and specifications in a manufacturing perspective. Also, non-geometric data such as surface texture specifications finish requirements, process notes, material specifications and welding symbols. [11]

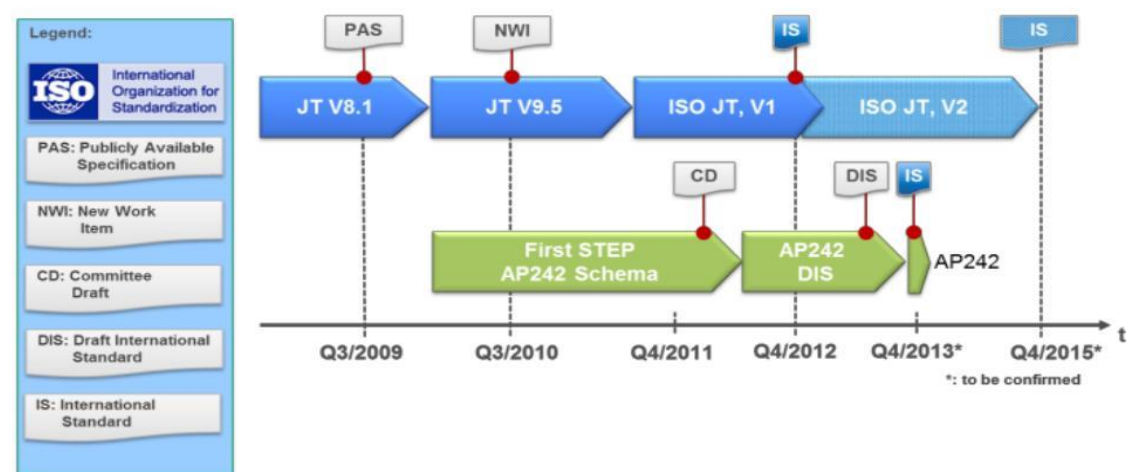

Fig. 1. ISO Roadmap for JT and STEP AP242. [1]

\subsection{Features of 3D file formats}

Different CAD systems have different ways of representing a CAD model, which is determined by the system's modelling kernel. Some CAD systems use the same modelling kernel as other systems. For example, ACIS by Spatial Technology Corporation 
and Parasolid by Siemens. Other companies use completely proprietary modelling kernels that are not shared. The modelling kernel determines how the CAD model is mathematically represented, semantically represented, and the internal accuracy of the geometric definitions. [12]

The issue of legacy information have brought about interoperable and neutral (lightweight) file formats into existence. The problems between those formats are mainly perceived when we look at the features (the shape, the appearance and the scene) of the models in each neutral file format and also the PMI.

- The shape of a model is often stored as a set of 3D points or vertices. The surfaces are stored as a series of polygons or faces that are constructed by indexing these vertices. This kind of format is known as tessellated.. If truly smooth surfaces are required, at any scale, then a convenient option is the use of Non-Uniform Rational B-Spline patches (or NURBS). These parametric surfaces are made up of a relatively small number of weighted control points and a set of parameters known as knots. BRep provides explicit representations for the geometrical elements such as vertexes, edges, faces, etc. Designing a shape can be done along the lines of constructive solid geometry (CSG) that uses Boolean operations on simple shape primitives such as cubes, cylinders, spheres, etc.

- The appearance, in its most common form materials entails applying an image or texture to the surface of the model. A model that does this must store these texture coordinates within the 3D data file. Most 3D file format support texture mapping. There would be three dimensions for three-color components corresponding to color spaces, such as RGB. Many file formats are supported storing material properties. However, an application that loads material properties usually ignores many of these properties when a user is manipulating the object.

- The scene describes the layout of the 3D model in terms of cameras, light sources, and other nearby 3D models. The camera is defined by some parameters as magnification and principal point, location, the direction the camera is facing and an arrow indicating which direction is up. Different formats for sharing the model.

\section{Case study}

Due to the ephemeral nature of CAD file formats and the applications that work with them, the migration of CAD information into lightweight formats could be a solution for preserving, exchanging and recovering information in the future. So, the need to correctly visualize the model, access the geometric and topologic information and geometric definitions and tolerances, entails the analysis and choice of some standard formats, but also the answers to: Which format are we going to choose? Which free viewers are available? Have the formats been recognized by some standard organization? What about the data exchange and the visualization?

Based on the formats supported by the community, those used by most of the companies and the CAD packages and third party software available to our use, we selected STEP, JT, 3D PDF, and STL in order to notice their discrepancies with respect to some criteria chosen. 
The chosen criteria are:

- The size: Since we are dealing with the so-called lightweight file format, the size is an important criteria for checking which format less size than other. This criterion could affect the opening of the model. A file less size than another one could take less time in a PDM.

- $\quad P M I$ : It includes the geometric dimensioning and tolerancing, which is used to communicate permissible deviations of product. It provided by CAD can describe dimensional tolerances on length and diameter, and geometric tolerances on flatness, perpendicularity, position, surface profile, and circular out [13]

- Documentation : Does It exist a document oriented structured able to containt 3D model and access the PMI

- Data exchange : Companies, in-house, want to be more collaborative. The standardized methods aim to improve the performance of data exchange. The standardized formats can be used to convert CAD to layers for building parts.

- Visualization: Given that we are translating the file format from one format to another, it is important to understand if some features are missing, if the parts' names are the same. The PMI is visible or not. Which file format does not allow the tree view's components (geometry and topology) representation?

\subsection{Software and hardware}

CATIA from Dassault Systèmes and CREO from PTC were used in order to perform the test. CrossManager (dkt) software from DATAKIT was used to convert legacy CAD file format to STEP, STL, JT and 3D PDF. CAD Assistant from Open Cascade was used to analyze the completeness of the interoperable file formats and the integrity of all the CAD models. It could not read file formats as STL, 3DPDF at the time of testing. So, the tested file formats were STEP and JT. The results were the measures of the volume, surface. For visualizing 3D PDF, we used adobe reader and STL the "window 3D viewer."

\subsection{Survey data Analysis}
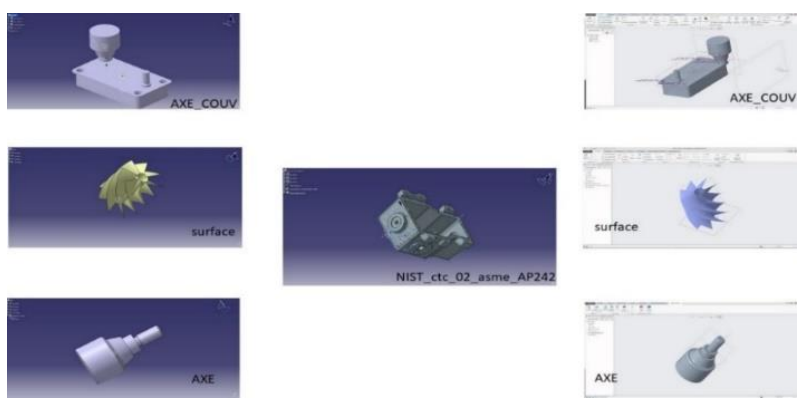

Fig. 2. Set of models used. 
Figure 2 shows the set of models used. The approach was, by taking one model designed in CATIA and CREO with the same characteristics, same features ( everything the same) and converted to STEP, STL, 3D PDF and JT formats through the means of CrossManager, what could be the difference in terms of the chosen criteria? Are the file format more influenced by the translation process or by the legacy CAD systems?

NIST_ctc_02_asme1_ap242 for observing the discrepancies with respect to the same model translated to other formats.

Figures 3 shows the synopsis of the translations process. AXE_COUV represents an assembly model. Surface is a surface part. The same models are represented in CATIA and CREO in order to be analyzed and to observe the differences.

Cat_dkt_stp and Prt_dkt_stp are the STEP file format got using directly the translation of the legacy file format through Crossmanager.

Cat_dkt_JT and Prt_dkt_JT are the JT file format got using directly the translation of the legacy file format through Crossmanager.

NIST_ctc_02asme1_ap242_JT is the original file format from NIST translated in JT.

Cat_dkt_PDF and Prt_dkt_PDF are the PDF file format got using directly the translation of the legacy file format through Crossmanager.

Cat_dkt_STL and Prt_dkt_STL are the STL file format got using directly the translation of the legacy file format through Crossmanager.

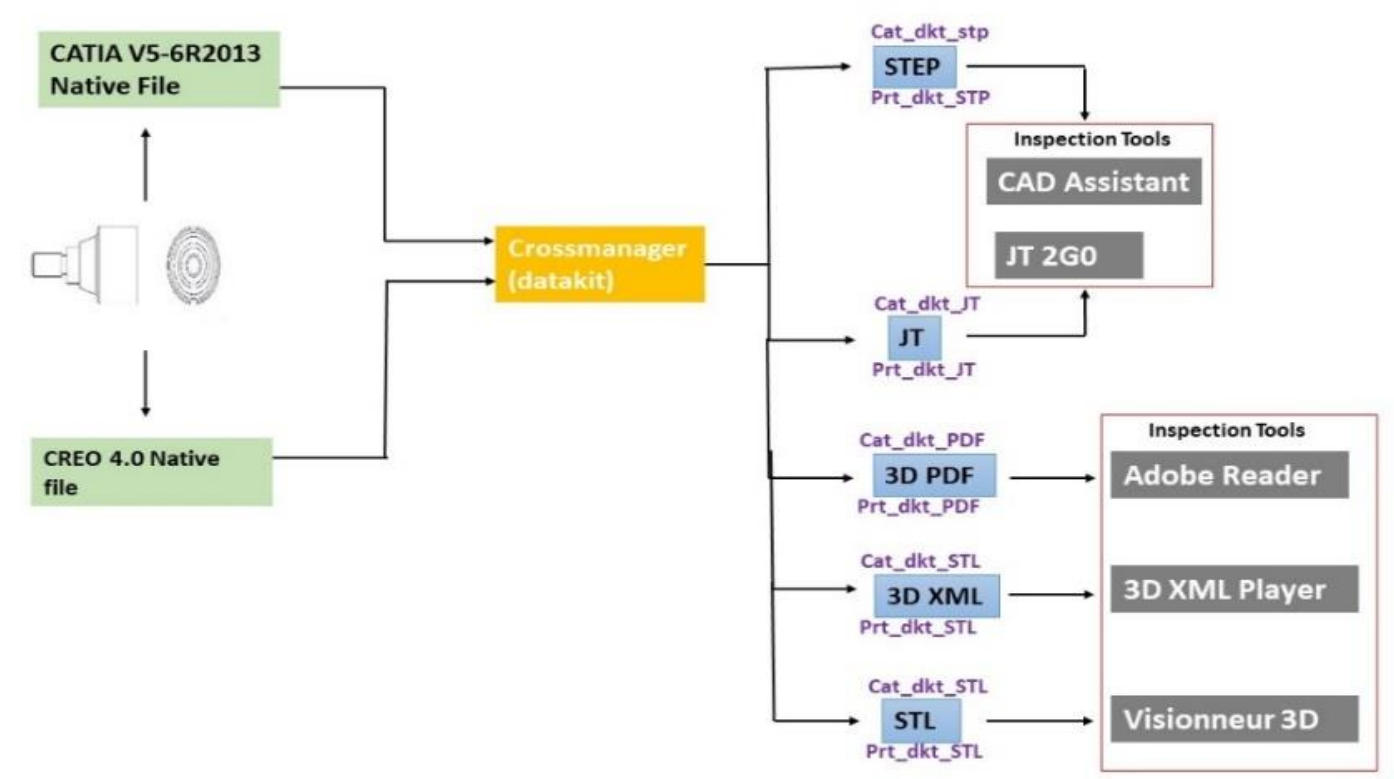

Fig. 3. Direct translation from legacy files format to another through Crossmanager 
The file formats were analyzed and compared. The data analysis was based on the preservation of information. The results were compared to each other in various ways to help determine possible trends. If changes occurred in models between translation processes, then those changes were determined using percent change where applicable. Percent change was determined by the relative change between the attribute of the newly translated model and the original attribute of the model.

Regarding the area and the volume, the comparison was just performed using STEP and JT thanks to the information obtained in CAD assistant. Therefore, we have compared the area of legacy file format with respect to JT and STEP by doing:

Delta_Area $=($ Area of the translated file format / Area of the legacy file format $) * 100$

Delta_Volume $=($ volume of the translated file format $/$ volume of the legacy file format) $* 100$

During our analysis, we have observed an increase of the model's areas from CREO during the translation process to other formats, while those from CATIA decrease their areas in comparison to the original ones. The volumes of the STEP files are almost equal to the legacy formats while those from JT are a little bit different. The difference regarding the volume and the area is around $4 \%$ on average. Consequently, we have a slight difference between regions and volume of objects in the two files.

Table 1. Translation path material property preservation

\begin{tabular}{|l|l|l|l|}
\hline & material name & Colour & Density \\
\hline From Cat or Prt to STP & yes & yes & No \\
\hline From Cat or Prt to JT & Yes & yes & No \\
\hline From STP to JT & yes & yes & No \\
\hline From JT to STP & Yes & yes & No \\
\hline
\end{tabular}

\subsection{Results assessment}

\section{$\underline{\mathrm{PMI}}$}

STEP AP242 and JT integrate table 1 and cover many computable representations for several types of 3D model data, including geometric definition and tolerances. The STEP and JT characteristics allow 3D PMI module to represent product information that is machine-readable. The module uses XML and EXPRESS schema languages to define product data model. CAD assistant shows the material converted in STEP and JT. In addition, the color of the part is visible. STL is dedicated to printing format, which has been used to transfer information from CAD software to printing hardware. The STEP and JT file keep the characteristics of precise geometry, tessellated geometry and tolerances as we can see in figures 4 -a and figures 4-b. 
Additional features of lightweight formats not part of STEP specification and 3D PDF, as advanced material lighting properties, level of detail mechanism, some level of compression or many STEP features not available in the lightweight formats as from features, construction history drafting capabilities. Some possibly features, not available by default need to be extended manually in the GT2Go. Consequently, JT and STEP are suited for this task.

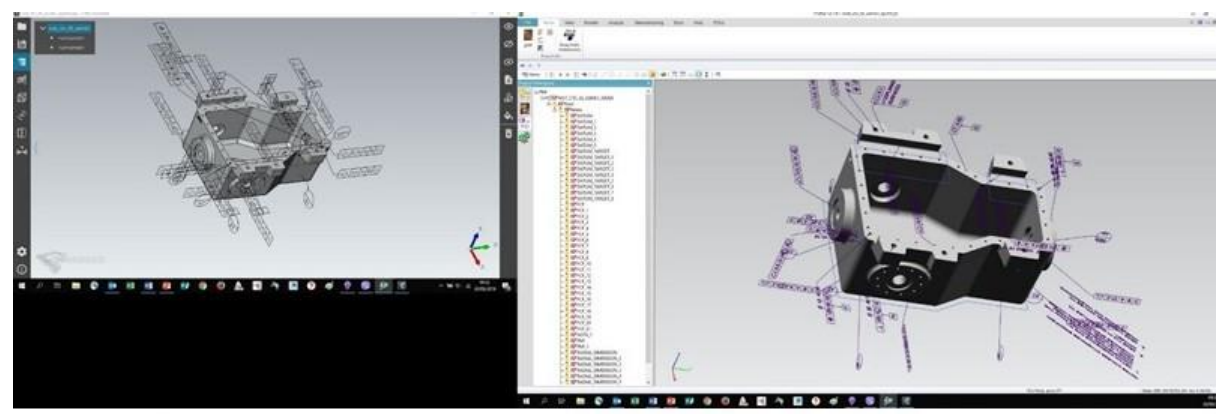

a)

b)

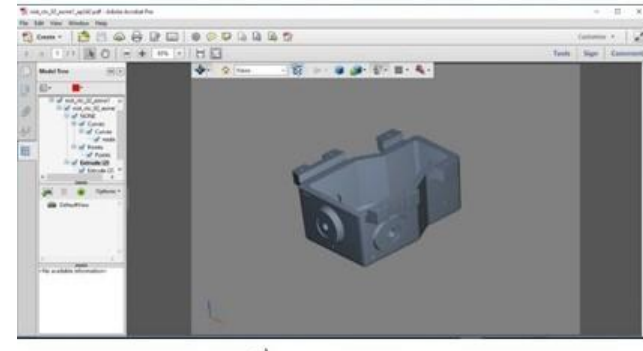

c)

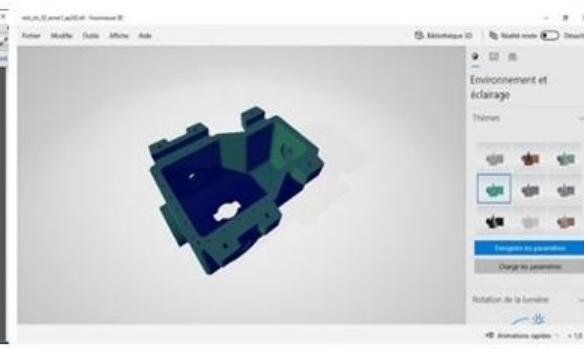

d)

Fig. 4. NIST model in a) STP; b) JT; c) 3D PDF; d) STL

\section{$\underline{\text { SIZE }}$}

Regarding the file size of the different models, the test results were found using the file size of the legacy CAD. STEP and STL are the ones much sized and JT file sizes are the lowest in most cases. By doing some calculations with respect to our samples, JT files are almost $60 \%$ lighter than STEP files and is the best one in terms of size. The size of the data is determined by the data content instead of the format itself. The fact that JT is less size that another format could make JT the ideal candidate suited in a PDM system because its opening could be faster than the others.

\section{DOCUMENTATION}

Because of its document-oriented structures, 3D PDF is an excellent solution for this purpose and any device that allows adobe reader can read it. STEP and JT can also be suited for this since they have been standardized ISO, so they provide a very good container for 3D models. 
With respect to PLM context, 3D PDF is the most suited because it allows 3D information to be represented together with other information. All the data are contained in a single document or file.

\section{DATA EXCHANGE}

Because of the numerous applications, STEP seems best suited for that since it matured long time ago. JT is also suited to exchange information, but compared to STEP, it needs to mature. Regarding 3D PDF or STL, because of the lack of exact representation and the lack of some information they are not suitable. It provides implementation methods for data exchange

\section{VISUALIZATION}

With the translation of files through Crossmanager, we can obtain either a faceted or a tessellated representation. In addition, the capacities of the available viewers make JT (figures 4-b) and 3D PDF (figures 4-c) ideal for visualization. In fact, with JT and 3D PDF, we can notice in the tree view, the parts forming the assembly of the legacy file translated, while with STEP, it is not possible.

In terms of metadata, all elements constituent the assembly structure in these formats are displayed at the level of the tree view regardless the viewer. So, the metadata are visible. The name of the shares (parts, sub-parts, assembly etc.) is as described in the native CAD system. Consequently, JT is suited for the visualization case thanks to the access to the geometry, topology and PMI

\section{CONCLUSION}

Due to the examination methodology selected for this study, we used CAD systems CATIA and CREO and third party software CAD assistant and JT2Go. After analyzing the different 3D neutral formats, it is clear that depending on the specific use case, one file format could have an advantage over another. However, given the interest which our study has shown in focusing on the geometric and topologic information and reading of PMI (color, material, tolerances, etc.), it could be said that the JT file is very promising. Because, despite the translations from one file format to JT file format, it is always possible to access the PMI correctly and visualize the geometric and topologic information. The tree view of JT neutral file format is done unambiguously, with a clear representation of the components.

\section{REFERENCES}

1. A. Katzenbach, S. Handschuh, and S. Vettermann, "JT format (ISO 14306) and AP 242 (ISO 10303): The step to the next generation collaborative product creation," in IFIP Advances in Information and Communication Technology, 2013, vol. 411, no. Iso 14306, pp. 41-52.

2. W. Solihin, C. Eastman, and Y. Lee, "Multiple representation approach to achieve highperformance spatial queries of 3D BIM data using a relational database," Autom. Constr., vol. 81, pp. 369-388, Sep. 2017. 
3. S. Terzi, A. Bouras, D. Dutta, M. Garetti, and D. Kiritsis, "Product lifecycle management: from its history to its new role," Int. J. Prod. Lifecycle Manag., vol. 4, no. 4, p. 360, 2010.

4. W. Zhu, M. Bricogne, A. Durupt, S. Remy, B. Li, and B. Eynard, "Implementations of Model Based Definition and Product Lifecycle Management Technologies: a Case Study in Chinese Aeronautical Industry," IFAC-PapersOnLine, vol. 49, no. 12, pp. 485-490, 2016.

5. K. McHenry and P. Bajcsy, "An overview of 3D data content, file formats and viewers," Technical report: National Center for Supercomputing Applications, 2008.

6. G. Tassey, D. Ph, S. A. Martin, and S. A. Martin, "Interoperability Cost Analysis of the U . S . Automotive Supply Chain," Final report: Research Triangle Institute Final Report. RTI Project, 1999.

7. A. Ball, L. Ding, and M. Patel, "Lightweight Formats for Product Model Data Exchange and Preservation," PV 2007 Conf. Oberpfaffenhofen, Ger. Oct. 9-11, no. 1, pp. 1-7, 2007.

8. N. W. Hartman, D. Ed, and A. Lim, "A Comparison of Functionality between STEP AP 203 E2, 3DXML, JT, and U3D Technologies," 3D Collab. interoperability, 2008.

9. PROSTEP AG, "3D Formats in the Field of Engineering - a Comparison," p. www.prostep.com.

10. J. Boy, P. Rosché, G. Hirel, and J. Haenisch, "Recommended Practices for AP242 Business Object Model XML Assembly Structure," CAx-IF Recomm. Pract., pp. 1-184, 2015.

11. J. Xiao, B. Eynard, N. Anwer, J. Le-Duigou, \& A. Durupt. " Information exchange standards for design, tolerancing and Additive Manufacturing: a research review ", International Journal on Interactive Design and Manufacturing, vol. 12, no.2, pp.495-504, 2018, Doi: 10.1007/s12008-017-0401-4

12. S. Gerbino, "Tools for the interoperability among CAD systems," in Proceedings of ADMINGEGRAF International Conference on Tools and Methods Evolution in Engineering Design, 2003.

13. R. Lipman, \& J. Lubell. "Conformance checking of PMI representation in CAD model STEP data exchange files". Computer Aided Design, vol. 66, pp. 14-23, Doi: 10.1016/j.cad.2015.04.0022012. 